\section{Category}

Synthesis of

Materials and

Unnatural Products

\section{Key words}

sumanene

fullerene fragment

bowl-shaped

compounds

\section{Synfact}

H. SAKURAI*, T. DAIKO, T. HIRAO* (OSAKA UNIVERSITY, JAPAN)

A Synthesis of Sumanene, a Fullerene Fragment

Science 2003, 301, 1878-1878.

\title{
Synthesis of Sumanene
}
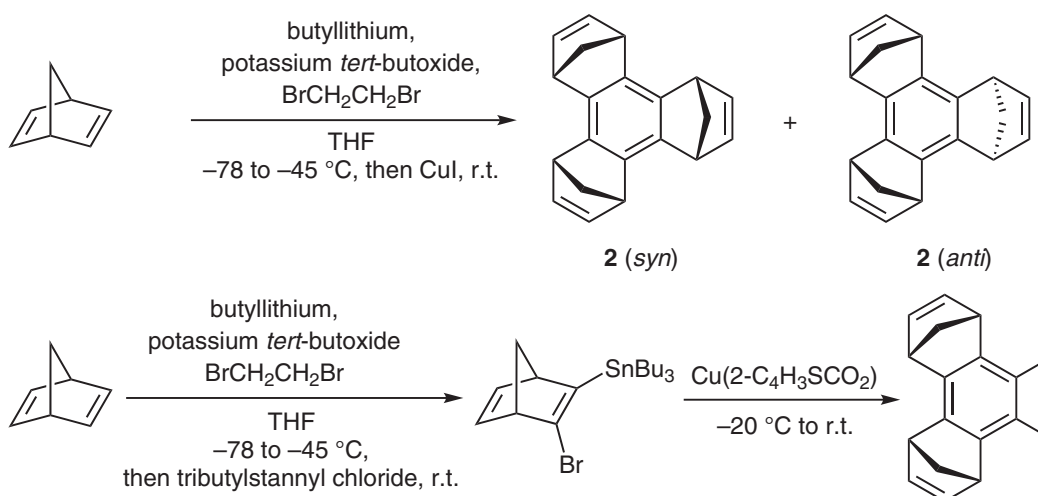

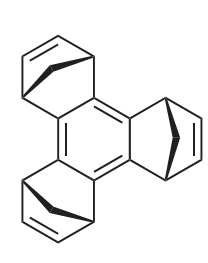

$2(s y n)$
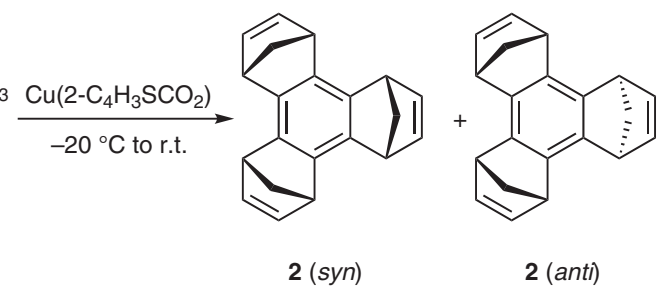

2 (anti)<smiles>CCCCC1=C(Br)C2C=CC1C2</smiles>

2 (syn)
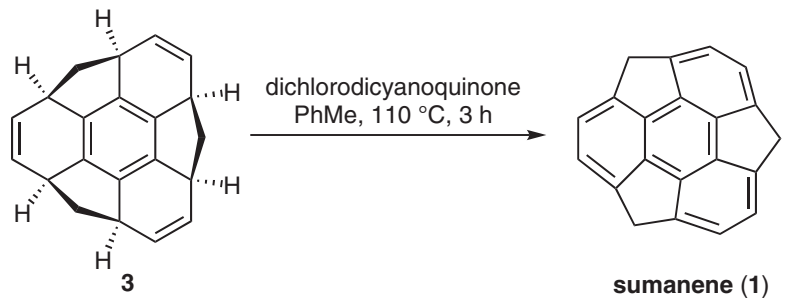

sumanene $(1)$
Significance: As model compounds of fullerenes as well as possible synthetic intermediates for artificially designed fullerene derivatives, bowl-shaped $\pi$-conjugated compounds become important. A key partial $C_{3 v}$-symmetric structure of fullerenes, sumanene, is synthesized in this paper. Sumanene has advantages that allow it to undergo further functionalization to create new bowl-shaped molecules. The authors report a synthetic method that enables access to sumanene under mild conditions in a short series of steps that start from commercially available norbornadiene.
Comment: In this paper, the authors present an efficient strategy to synthesize sumanene. Instead of starting from the planar compounds, the authors constructed the three-dimensional framework by using tetrahedral $\mathrm{sp}^{3}$ carbon units. Two procedures were tried to achieve the trimerization of norbornadiene, and the stepwise transmetalation via an organotin compound increased the overall yield of syn-2 from $2 \%$ to $11 \%$. In contrast to the other wellstudied bowl-shaped compound corannulene, sumanene is shown to be very rigid and the bowl-tobowl inversion only occurs at high temperature. 